COMMENT. Incomplete spinal cord injury and Brown-Sequard syndrome are adult disorders, but motor vehicle accidents and violence may be responsible in children. The authors cite 3 additional pediatric cases reported in the literature. High-dose steroids in 2 cases (including the present case) and surgery in 2 resulted in good to complete recovery.

\title{
POSTCONCUSSIVE SYMPTOMS AND NEUROCOGNITIVE FUNCTION AFTER MILD TBI
}

The frequency, nature, and recovery from postconcussive symptoms (PCSs) after mild traumatic brain injury (MTBIs) are described in 28 patients, 10 to 17 years of age, and their neurocognitive function in the initial 4 to 5 weeks are compared with 45 injured controls without MTBIs, in a prospective, longitudinal study at University of Michigan, Arn Arbor, MI. MTBI patients reported significantly more PCSs than controls, and these persisted for weeks after injury but decreased significantly between 1 and 4 to 5 weeks. Symptoms included altered mental status or confusion in 11 (39\%), dizziness in 23 $(82 \%)$, nausea/vomiting in $19(68 \%)$, incoordination (9), blurred vision (7), diplopia (4), and irritability in $3(11 \%)$. Posttraumatic amnesia in $8(29 \%)$ at baseline was not associated with an increase in PCSs or poorer neurocognitive function; trends during follow-up were identical for MTBI participants with and without amnesia. Patterns of recovery following MTBIs on a neurocognitive Trail-Making Test Part B differed significantly from controls, whereas other neurocognitive measures were not different. PCSs and neurocognitive deficits occur both in patients with MTBIs and in children with injuries other than brain trauma, but the symptoms are milder in the non-TBI patients and their recovery patterns are different on the Trail-Making Test Part B. (Sroufe NS, Fuller DS, West BT, Singal BM, Warschausky SA, Maio RF. Postconcussive symptoms and neurocognitive function after mild traumatic brain injury in children. Pediatrics June 2010;125:e1331-e1339). (Respond: Nicole S Sroufe MD, MPH, 1500 E Medical Center Dr, Room TC B1 380, Ann Arbor, MI 48109. E-mail: nicolel@med.umich.edu).

COMMENT. Postconcussive symptoms decrease between 1 and 4 to 5 weeks after mild traumatic brain injury in children, and neurocognitive deficits resolve by 3 to 6 months after injury in the majority. Posttraumatic amnesia does not predict development of postconcussive symptoms or neurocognitive dysfunction. Children with injury other than traumatic brain injury may also have postconcussive-like symptoms and neurocognitive deficits but these are milder and recovery patterns are different.

A recent questionnaire, follow-up study (average 2 years after trauma) of children with head trauma (Glasgow Coma Score $<$ or $=8$ ) in 38 families found persistence of paresis $(16 \%)$, cranial nerve damage $(13 \%)$, incontinence $(5 \%)$, or incoordination (18\%). Mental and cognitive problems were frequent, even in children with light head trauma and often manifested after release from hospital. Inferior performance in school in $26 \%$ was associated with frequent absences, and social withdrawal and aggressive behavior led to family tensions. (Kapapa T et al. J Child Neurol April 2010;25:409-422). Children with minor head trauma may have functional behavioral morbidity that reflects parental overreaction despite rarity of physical sequelae. (Casey R et al. Pediatrics 1986;78:497). 